\title{
First Edoardo Amaldi Conference \\ on \\ Gravitational Wave Experiments
}

Villa Tuscolana, Frascati (Rome), 14-17 June, 1994

This conference is intended to honour Edoardo Amaldi for his role in promoting and actively participating in gravitational wave research. Its purpose of this conference is to bring together scientists engaged in research both with gravitational wave antennas and with neutrino telescopes, with special emphasis on recent developments in resonant cryogenic antennas.

The topics to be covered are:

Gravitational wave experiments with resonant mass, interferometers and space detectors

Neutrino telescopes

Astrophysical sources

Coincidences among different detectors

The deadline for registration and room reservation is April 15, 1994.

For more information please contact:

Liù Catena

Departimento di Fisica

Università di Roma "Tor Vergata"

Via della Ricerca Scientifica 1

1-00133 Rome, Italy

Tel: +39672594301

Fax: +396.2023507

E-mail: Catena@roma2.infn.it

Decnet: 39992::Catena

Silvia Giomini

INFN-LNF, via Enrico Fermi 40

I-00044 Frascati (Rome), Italy

Tel:.+3969403643

Fax: +3969403243

E-mail: Silvia@irmlnf.bitnet

Decnet: Vaxlnf::silvia 